\title{
Diferencias entre Cysticercus cellulosae y C. racemosus
}

\author{
Francisco Biagi F.* por $\quad$ Carlos E. Briceño* \\ y
}

Bertha Martínez*

(Recibido para su publicación el 9 de mayo de 1961)

En el hombre se encuentran dos formas de cisticercos y desde el siglo pasado se acepta que ambas son formas larvarias de Taenia solium (1, 5, 9). Sin embargo, estas dos formas larvarias presentan diferencias morfológicas, serológicas y anatomopatológicas; en el presente trabajo expondremos estas diferencias, aprovechando material de 97 autopsias con cisticercosis (3).

\section{MORFOLOGIA.}

Cysticercus cellulosae se caracteriza por tener forma redonda u ovoide, de 3 a $18 \mathrm{~mm}$ de diámetro y por estar constituído por una membrana delgada, que circunscribe una cavidad llena de líquido. En cierto lugar, esta membrana se encuentra plegada hacia adentro, dando origen a un cuello que en su extremo tiene el escólex con 4 ventosas y una doble corona de 24 a 32 ganchos; los ganchos pequeños miden de 110 a $140 \mu$ de longitud, y los grandes, de 160 a $180 \mu$.

Estas características se observan a fresco con facilidad al microscopio, comprimiendo el parásito entre dos portaobjetos, pero son difíciles de precisar en los cortes histológicos. En el parásito vivo se pueden observar sus movimientos, o al menos se observan con nitidez los pliegues del cuello y no se desprenden de su implantación las ventosas y los ganchos. Lo contrario se observa cuando el parásito ha muerto algún tiempo antes de su fijación u observación, y además suelen encontrarse masas blancas de consistencia pastosa en el interior del quiste (fig. 1).

* Sección de Parasitología, Unidad de Patología, Facultad de Medicina; U.N.A.M. Hospital General, México 7, D. F. 
En los cortes histológicos, la membrana se encuentra formada por una cutícula externa, a continuación una capa celular con gran cantidad de núcleos y en la porción interna una capa reticulofibrilar. La membrana suele ser de grosor uniforme; solo en una ocasión hemos observado "cilios" en la superficie exterior (fig. 6), lo cual consideramos como hallazgo excepcional.

El cuello, en su implantación tiene una morfología histológica muy semejante a la membrana, pero va modificándose hasta mostrar una cutícula gruesa más interna y plegada, seguida de la capa celular que además de los núcleos contiene gran cantidad de corpúsculos ovoides, y finalmente la porción reticulo-fibrilar (fig. 2).

En el extremo del cuello, en algunos cortes, se encuentran las ventosas con sus fibras musculares radiadas y ocasionalmente fragmentos de ganchos de color amarillo refringente (fig. 3 ).

En los parásitos viables se observan las estructuras con nitidez. En cambio, cuando el parásito ha muerto algún tiempo antes de su fijación, se pierde la nitidez observando porciones amorfas y eosinófilas; las ventosas conservan su morfología durante más tiempo, así como los ganchos. Además cuando el parásito ha muerto, estimula la producción de reacción inflamatoria a su alrededor.

Diversos autores han descrito minuciosamente algunos aspectos morfológicos de estos parásitos $(4,10,11,12)$.

Cysticercus racemosus se caracteriza fundamentalmente por carecer de escólex. Hemos observado ejemplares que miden desde 5 a $90 \mathrm{~mm}$ de diámetro. Su membrana es delgada, macro y microscópicamente semejante a la de C. cellulosae y circunscribe una cavidad generalmente irregular, pues presenta lobulaciones (fig. 4); no hay tabicación de la cavidad. En pocas ocasiones hemos encontrado $C$. racemosus pequeños y esferoidales, sin lobulaciones. En la superficie interna a veces se ven pequeñas excrecencias.

Histológicamente se diferencia de $C$. cellulosae porque presenta frecuentemente irregularidad en el espesor de la membrana o mamelones en su superficie exterior (fig. 5). Además de manera muy constante pueden apreciarse "cilios" en su superficie externa (fig. 6). Las excrecencias presentan morfología histológica variada.

A veces, en los cortes histológicos, se pueden encontrar las características morfológicas que permiten con certeza identificar el tipo de Cysticercus, pero esto se logra mejor estudiando el parásito in toto en el microscopio estereoscópico.

Al igual que $C$. cellulosae, cuando $C$. racemosus se encuentra viable, presenta sus estructuras histológicas bien nítidas y cuando ha muerto y se encuentran en involución, los cortes de la membrana se aprecia $a_{n}$ como bandas hialinizadas y eosinófilas, frecuentemente rodeados de reacción inflamatoria, células gigantes y masas de material amorfo.

Cisticercos Anormales. En nuestra serie de 97 autopsias de cisticercosis, hemos encontrado 5 parásitos que presentan morfología diferente de la descrita, y se encontraron en cadáveres distintos. Hay pocas referencias bibliográficas sobre cisticercos anormales (8). 
Tres parásitos alojados, uno en ventrículo lateral, otro en el fondo de la cisura de Silvio y otro en parénquima cerebral, presentaron morfología de $C$. cellulosae, encontrándose una malformación en su cuello. Macroscópicamente el cuello estaba muy engrosado en su base (fig. 7) y en los cortes, esta parte engrosada se vió constituída por tejido reticulofibrilar con muchas cavidades de tamaño variable.

Un parásito alojado en el pedúnculo cerebral izquierdo, presentaba una doble membrana (fig. 8). La membrana exterior conservaba su morfología de C. cellulosae pero circunscribía una cavidad de $30 \mathrm{~mm}$ de diámetro; la porción de la membrana que delimitaba la pequeña cavidad, carecía de cutícula. En el interior de la pequeña cavidad había un cuello y escólex semejante a los de C. cellulosae, pero tenía solamente 18 ganchos.

Otro parásito encontrado en la base del cerebro, presentaba también mortología de C. cellulosae, con escólex normal, sin embargo presentaba a los lados de su membrana otras dos cavidades quísticas adheridas, pero separadas de la principal por un tabique (fig. 9); parecía como si la membrana presenta. ra lobulaciones como C. racemosus, pero éstas estaban tabicadas, cosa que no ocurre en estos últimos parásitos.

Uno de los casos que presentó un parásito con cuello engrosado, tenía 96 cisticercos; otros 7 parásitos que se revisaron de la misma autopsia, presentaban morfología de C. cellulosae. La gran cantidad de cisticercos encontrados hace pensar que se trata de un caso de autoinfección. En el intestino de este cadáver se encontró un ejemplar de Taenia saginata. De ninguna manera podemos afirmar que este ejemplar de T. saginata hubiera originado la autoinfección, pero este hallazgo podría tener alguna relación con la existencia de un cisticerco anormal.

\section{SEROLOGIA}

En 1958 señalamos que no había reacción de precipitación cruzada en ambas formas de cisticercosis (2). En la actualidad hemos reunido mayor número de observaciones haciendo reacción de precipitación con antígenos de $C$. cellulosae y de $C$. racemoszis. Puede verse en el cuadro 1 que se presenta mucho mayor número de reacciones positivas con el cisticerco homólogo que con el heterólogo. Esto hace suponer que la composición antigénica de ambos parásitos es diferente y por lo tanto, es posible que pertenezcan a especies distintas de Taenia.

\section{ANATOMIA PATOLOGICA}

En el cuadro 2 puede apreciarse la frecuencia con que hemos encontrado los tipos de cisticerco en casos de autopsia; puede verse que en 7 ocasiones coexistían C. cellulosae y C. racemosus.

Como se señala en el cuadro $3, C$, cellulosae se encuentra principalmente en parénquima del sistema nervioso cent al, y en cambio C. racemosus se alo. 
ja en distintas cavidades, quedando libre de líquido céfaloraquídeo. Se ha dicho que cuando los cisticercos caen al líquido céfaloraquídeo estos se transforman en $C$. racemosus (6); no es fácil aceptar esta afirmación habiendo encontrado esta forma de parásitos en pleno parénquima; además, como ya se señaló, hemos observado C. racemoszıs ovoides, sin lobulaciones y apenas de $5 \mathrm{~mm}$ de diámetro; esto sugiere que los parásitos tienen su morfología propia desde su formación y su diferente localización podría estar relacionada a características propias de cada tipo de parásito.

\section{CUADRO 1}

Frecuencia de positividad de la reacción de precipitación con antigeno de cisticercos, según el tipo de parásito encontrado en los enfermos

\begin{tabular}{|c|c|c|c|c|}
\hline Personas con: & $\begin{array}{r}\text { A } \\
\text { C. } \\
\text { Casos } \\
\text { estudiados }\end{array}$ & Positivos & $\begin{array}{r}\text { A. } \\
\text { Casos } \\
\text { estudiados }\end{array}$ & Positivos \\
\hline $\begin{array}{l}\text { Cisticercosis } \\
\text { cellulosae. } \\
\text { Cisticercosis } \\
\text { racemosus. }\end{array}$ & 14 & 11 & 1 & 0 \\
\hline
\end{tabular}

CUADRO 2

Tipos de cisticercos encontrados.

78 casos adecuadamente estudiados

\begin{tabular}{c|c}
\hline Tipo & Númeto de casos \\
\hline cellulosae & 53 \\
racemosus & 18 \\
Ambos & 7 \\
\hline
\end{tabular}

\section{DISCUSION}

Por semejanza morfológica de C. cellulosae del cerdo con los parásitos encontrados en el hombre, se acepta que estos últimos también son formas larvarias de Taenia solium. Como en el hombre también se encuentra C. racemosus, se aceptó que esta es una malformación de C. cellulosae. Sin embargo nunca se ha demostrado objetivamente este hecho. 


\section{CUADRO 3}

Localización de los cisticercos segiin su tipo o "especie".

Alojados en:

Casos con

C. cellulosae

Casos con

C. racemosus

Distintas porciones de parénquima del sistema nervioso central.

Ventrículos y espacio subaracnoideo

41

11

25

\section{CUADRO 4}

Frecuencia de meningitis basal crónica en casos de cisticercosis, de acuerdo con el tipo de los parásitos

\begin{tabular}{l|c|c|c}
\hline \hline & $\begin{array}{c}\text { Casos } \\
\text { estudiados }\end{array}$ & $\begin{array}{c}\text { Con meningitis } \\
\text { basal crónica }\end{array}$ & $\%$ \\
\hline C. cellulosae & 36 & 5 & 13,9 \\
C. racemosus & 22 & 10 & 45,5 \\
\hline
\end{tabular}

\section{CUADRO 5}

Frecuencia de distintas manifestaciones clinicas de acuerdo con el tipo de cisticerco. encontrado, en 56 casos de cisticercosis cerebral.

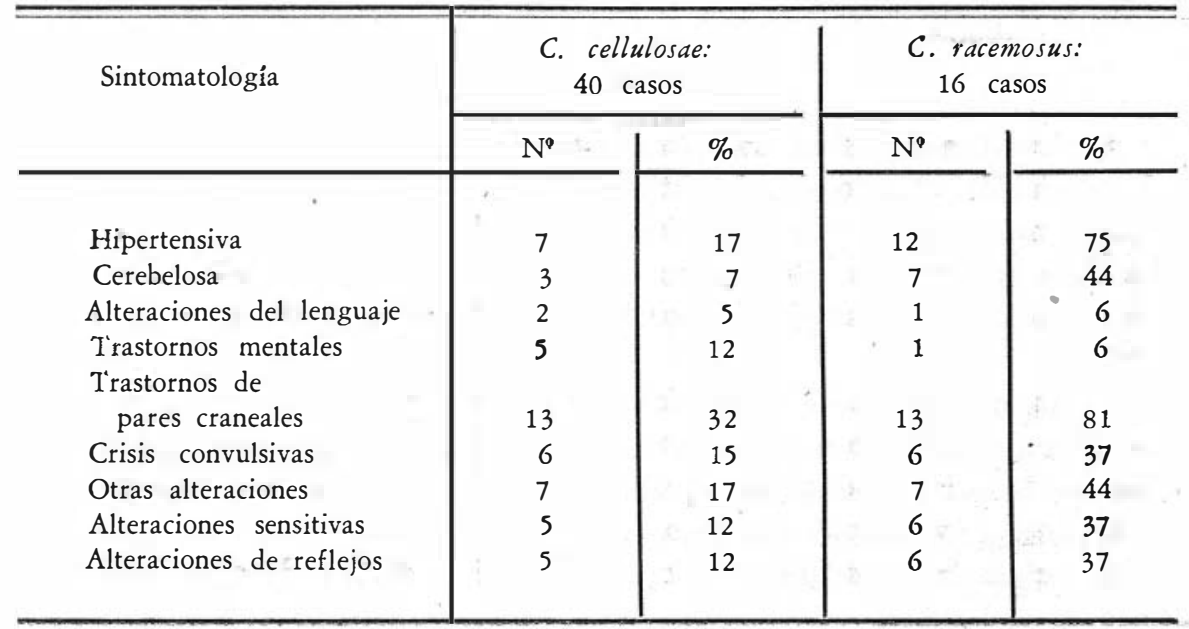


Como C. racemosus carece de escólex, no puede dar origen a parásitos adultos, de modo que no es fácil demostrar la identidad específica de estas formas larvarias.

La presencia de algunos C. cellulosae con morfología anormal, sugiere que estos parásitos eventualmente pudieran presentar morfología de C. racemosus. También apoya esta posibilidad el hecho de que en algunos casos coexisten ambos tipos de parásitos.

Sin embargo, existen 72 especies de Taenia de las cuales 12 se han encontrado en animales domésticos $(9,14)$. En México, al menos se ha reportado la existencia de $T$. taeniaeformis en el gato (7). De acuerdo con esto, el hombre puede estar expuesto a ingerir huevos de estas tenias y eventualmente, desarro. llar cisticercos diferentes de C. cellulosae. Creemos que C. racemosus bien podría ser un estado larval de una Taenia de algún animal doméstico, que al desarrollarse en el hombre origina un parásito sin escólex, probablemente por haberse alojado en un huésped anormal.

La existencia de $C$. racemosus en parénquima, o bien, mostrando ausencia de escólex desde que es pequeño y sin lobulaciones, hace pensar que no es un parásito que ha cambiado de morfología. La diferente localización en el hombre, también hace pensar en la dualidad de especies; quizá el argumento más interesante a este respecto son las diferencias serológicas encontradas.

Otro hecho que también al parecer apoya la dualidad de especies es que los cerdos infectados con C. cellulosae, cuando presentan cisticercosis cerebral, no se ha visto que desarrollen C. racemosus (13), sino exclusivamente parásitos con morfología de $C$. cellulosae.

Si estos dos tipos de cisticercos correspondieran a especies distintas de Taenia, probablemente su epidemiología sería diferente. Esto resulta muy importante desde el punto de vista de la prevención.

En todo caso el precisar el tipo de cisticercos es importante, pues como se vé en el cuadro 4 , los casos con $C$. racemosus presentan meningitis basal con mayor frecuencia y también presentan sintomatología más severa (cuadro 5). Es decir, desde el punto de vista clínico, la infección con $C$. racemosus es más grave.

\section{RESUMEN}

Se describen e ilustran Cysticercus cellulosae y C. racemosus aprovechando el material de 97 autopsias de cisticercosis. Se señalan las características diferenciales macroscópicas y microscópicas entre ambos tipos, así como las diferencias entre los parásitos viables o en involución, haciendo énfasis en la utilidad del estudio al microscopio estereoscópico. Se describen cuatro, C. cellulosae anormales.

Se anotan diferencias serológicas y anatomopatológicas encontradas en ambos tipos de cisticercosis y se discute la posibilidad de que estos parásitos sean formas larvarias de distintas especies de Taenia, lo cual es muy importante desde el punto de vista epidemiológico.

Se señala la importancia clínica de identificar el tipo de cisticerco encontrado en el hombre. 


\section{SUMMARY}

Descriptions and illustrations of Cysticercus cellulosae and C. racemosus are presented, from material obtained in 97 autopsies. Differential macroscopic and microscopic characters are pointed out, as well as differences in live and involution parasites. Value of study under stereoscopic microscope is emphasized. Four abnormal $C$. cellulosae are described.

Serologic and anatomo-pathologic differences found between both types of cysticercosis are noted, and the possibility is discussed that these parasites may be larval forms of different species of Taenia, which is important in epidemiology. The clinical importance of identifying the type of cysticercus found in man is pointed out.

\section{BIBLIOGRAFIA}

1. Bej.ding, D.^ L.

1952. Clinical Parasitology. 2" ed., 1139 pp., Appleton Century-Crofts, Inc., New York.

2. Biagi-F. F., y J. TAY

1958. A precipitation reaction for the diagnosis of cysticercosis Am. J. Trop. Med. Hyg., 7(1): 63-65.

3. Briceño, C. E., F. Biagi-F., y B. Martínez

1961. Observaciones sobre 97 casos de autopsia con cisticercosis. Prensa Med. Mex. (En prensa).

4 Cardoso, R. A. A.

1941. Diagnóstico histológico da cisticercose cerebral. Mem. Inst. Osw. Cruz, $36(1): 33-36$.

5. Craig, C. F. y E. C. Faust

1951. Parasitología clinica. Trad. $4^{a}$ ed. inglés, 882 pp., UTEHA, México.

6. EsCOBAR, A.

1952. Cisticercosis cerebral. Arch. Mex. Neurol. Psiquiat., 1(7): 149-167.

7. Flores BARROETA, L.

1955. Helmintos de los perros y gatos de la ciudad de México. An. Esc. Nac. Cienc. Biol:; 8(3.4): 159-202.

8. Huff, C. G. y P. C. BuCy.

1936. An abnormal Cysticercus cellulosae from a human brain. J. Parasitol., 22(1): 110 .

9. Neveu-Lemaire, $M$.

1936. Traité d'belminthologie médicale et véterinaire, 1514 pp., Vigot Fr., Paris.

10. Peralta, L.

1958. Contribución al conocimiento de la morfología macro y microscópica del cisticerco celuloso. Tesis, Esc. Biol., Fac. Ciencias UNAM. México. 
11. Ravens, J. R.

1955. Contribución a la histoparasitología de Cysticercus cellulosae y C. racemosus. Arch. Histol. Normal Patol., 6(1): 43-97.

12. Talice, R. V. y J. Gurri.

1949. Sur la morphologie du Cysticercus racemesus. Existence d'un revétement cilié sur sa paroi. Ann. Parasitol., 24(5-6): 412-413.

13. Thienpont, D. y Col.

1959. La cysticercose cérebral du porc. Ann. Soc. Belge Med. Trop., 39(4): 507-514.

14. YAMAGUTr, S.

1959. Systema Helminthum. Il. The cestodes of vertebrates. Interscience Publ. Co. New York.

Fig. 1: C. cellulosae en involución, mostrando una masa de material pastoso de color blanco en su interior.

Fig. 2: Porción de cuello de C. cellulosae. (obj. $40 \times)$.

Fig. 3: Corte de C. celluloscie mostrando una porción de ventosa con fibras radiadas, y fragmentos de ganchos (obj. $40 \times)$.

Fig. 4: Ejemplar de C. racemosus mostrando la ausencia de escólex y sus lobulaciones. 


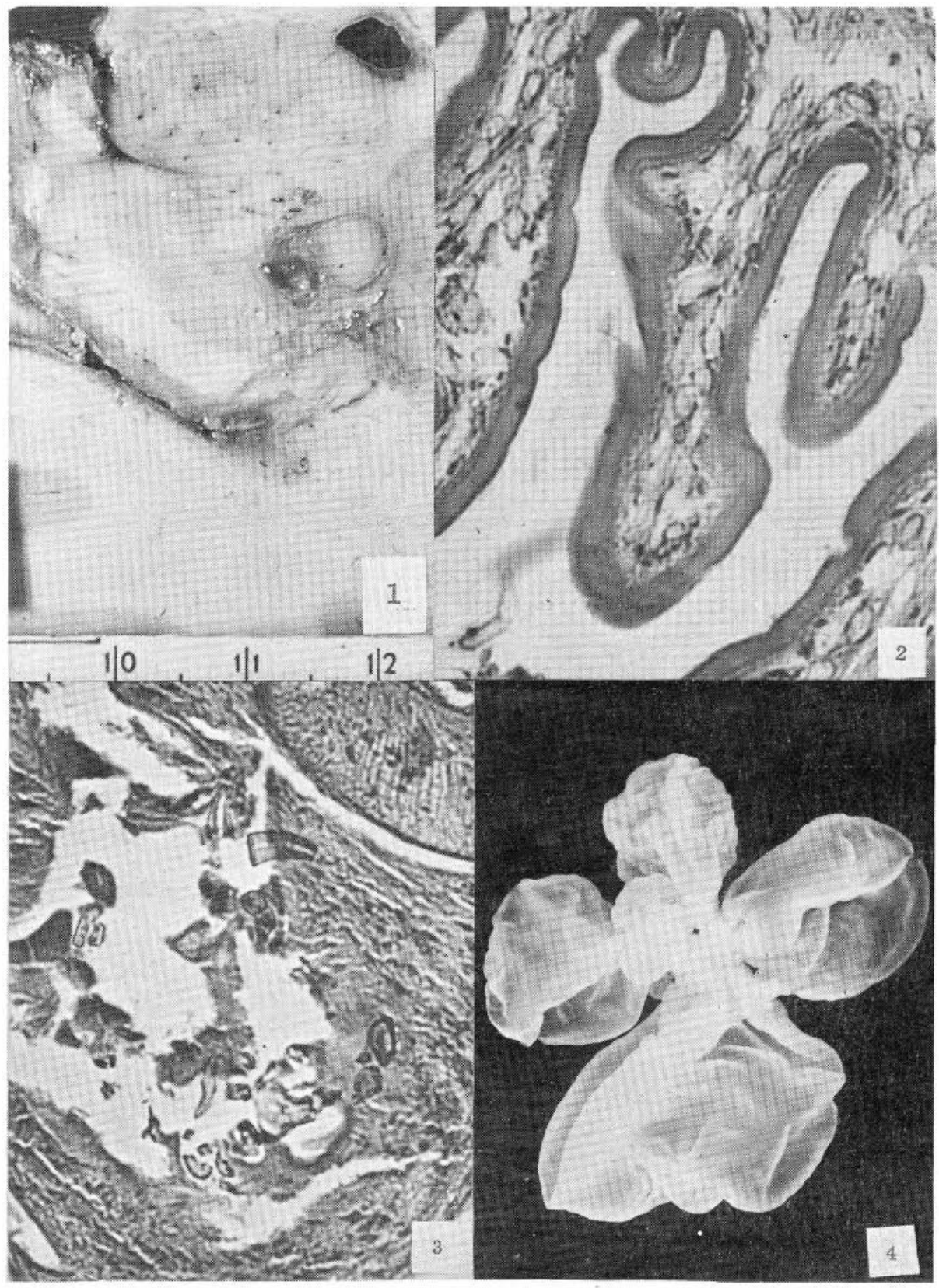


Fig. 5: Corte histológico mostrando mamelones en la superficie exterior de la membrana de $C$. racemosus (obj. $10 \times$ ).

Fig. 6: Membrana de C. racemosus mostrando "cilios" (obj. $100 \times)$.

Fig. 7: Cuello anormal, engrosado y con lobulaciones, de $C$. celluiosae.

Fig. 8: Cisticerco anormal, semejante a C. cellulosae, con una doble membrana.

Fig. 9: Cisticerco anormal, semejante a C. cellulosae, con dos lobulaciones tabicadas en su membrana. 


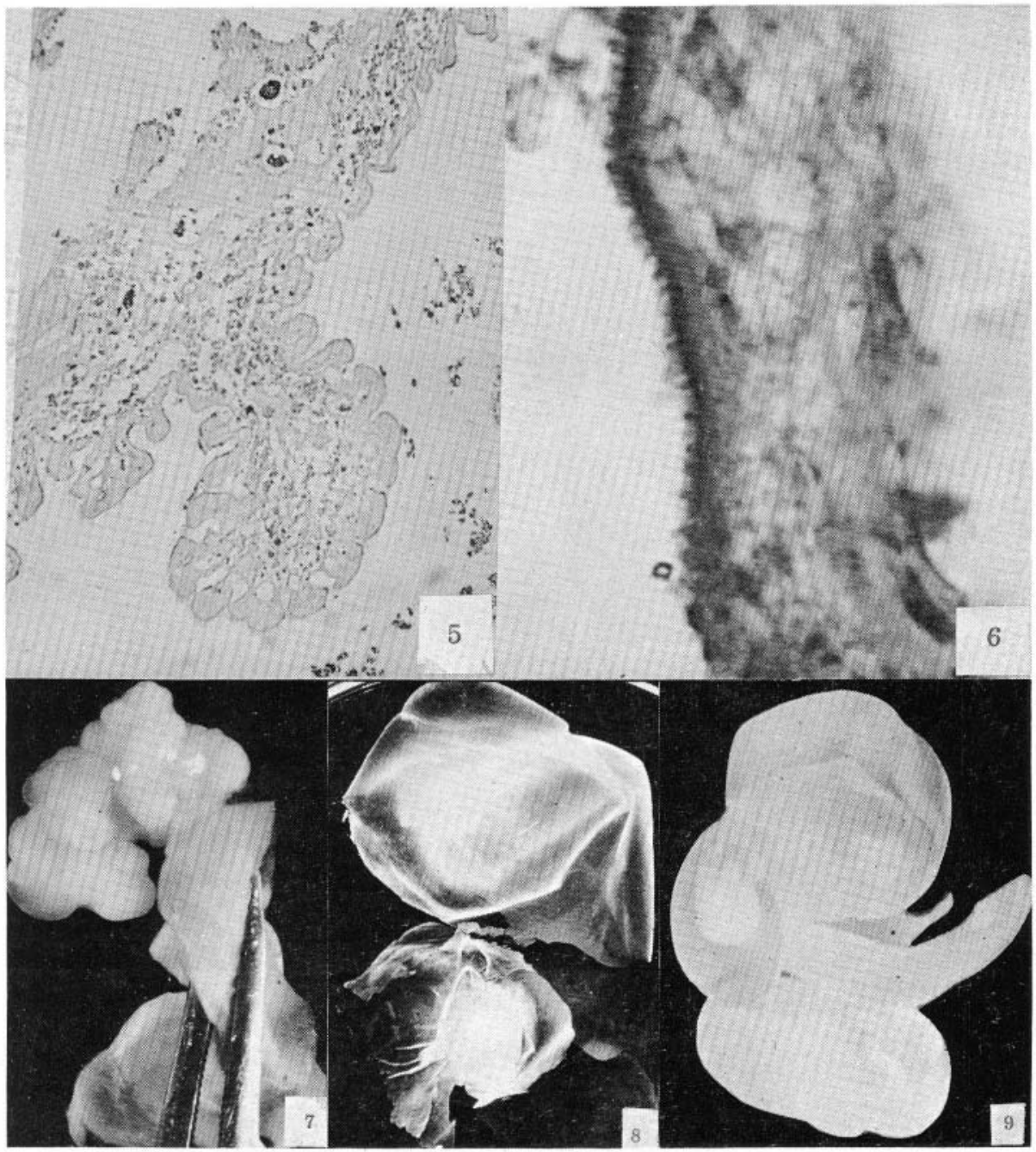

